\title{
Development of Virtual Emulation Modelling on the Reconfigurable Hot Forming Process and Its Digital Twin Implementation Perspectives
}

\author{
Belal M Aly ${ }^{\mathrm{a}, 1}$ and Kai Cheng ${ }^{\mathrm{b}}$ \\ ${ }^{a}$ Brunel University London
}

\begin{abstract}
In this paper, the development of virtual emulation modelling is presented on the reconfigurable hot forming process and its further implementation for the associated digital twins. When validating the developed Digital Twin system, it is essentially important to test the digital twin prior to its connection to a real physical asset especially from a safety and efficiency prospective. The development is focused on digital virtual emulation of the reconfigurable hot forming process, which can emulate the physical element as the means of validating the digital twin system with the throughout-digital virtual simulations and underlying results.
\end{abstract}

Keywords. Reconfigurable, Hot Forming, Die, Digital Twin, Industry 4.0, Virtual Modelling, Emulation Model.

\section{Introduction}

Digital Twin is regarded as one of the smart manufacturing futures. The adopted process for this research was the Re-configurable hot forming process with a focus on its virtual modelling and emulation, which provide the foundation further for its Digital Twin implementation aiming to solve the exact issues in the hot forming process, including:

- $\quad$ High reject rate

- High inefficiency

- $\quad$ Lack of the process predictivity and repeatability

- $\quad$ High prototyping costs

- $\quad$ High tooling cost

- Heavily manual oriented process

Furthermore, digital twin offers the promise to fully automate the manufacturing process from start to finish, full predictivity and repeatability of the process, to overcome inefficiency. The associated constant live optimization to reduce defects, simulation to reduce physical prototyping and the reconfigurable element of the system will reduce the tooling cost and cope with the increasingly challenging needs of small batch and large variety in modern manufacturing.

\footnotetext{
${ }^{1}$ Belal M Aly, Kai Cheng, Brunel University London, Uxbridge, UB8 3PH, United Kingdom;

E-mail: Belal.MohamedAly@brunel.ac.uk; Kai.Cheng@brunel.ac.uk
} 
Part of the digital twin system is a simulation subsystem that is responsible for taking data from the physical operations and running numerical simulations to predict and optimize performance. In this paper, we present the development of a like for like virtual emulation model to behave and act exactly like the physical machine. The virtual model aims to emulate the physical entity and to provide data and interact with the digital twin as if it is in physical operations. This provides the engineers the ability to test the digital twin system before real life implementation as well as the visual/data representation to manufacturers, exactly what the digital twin is able to deliver if implemented in their plan. The development uses Simumatik3D environment to emulate the hot forming process.

\section{Fundamentals Formulation}

Emulation models are frequently utilised as a substitute of a physical system, this comes with their own unique challenges. The emulation model is only ever as good as the data programmed into the model; thus, it is critical to ensure the data and behavior of the system lines up with that of a real physical world [4]. The data the emulation model will be providing to the digital twin system will be in the form of:

- Temperature

- $\quad$ Pressure of dies

- $\quad$ Measurement data of produced product

- $\quad$ Processing time

Figure 1 illustrates the connection and communication between the physical element and the digital twin. Temperature data is provided through infrared heat sensor cameras, pressure of dies is measured using the feedback from the actuating hydraulics and scanning cameras that capture the velocity of the moving dies. Processing time are measured from the start of the manufacturing process to the completion of the final product. As to measurement data of the produced product a $3 \mathrm{D}$ scanning camera is utilised to measure the final product against the CATIA model.

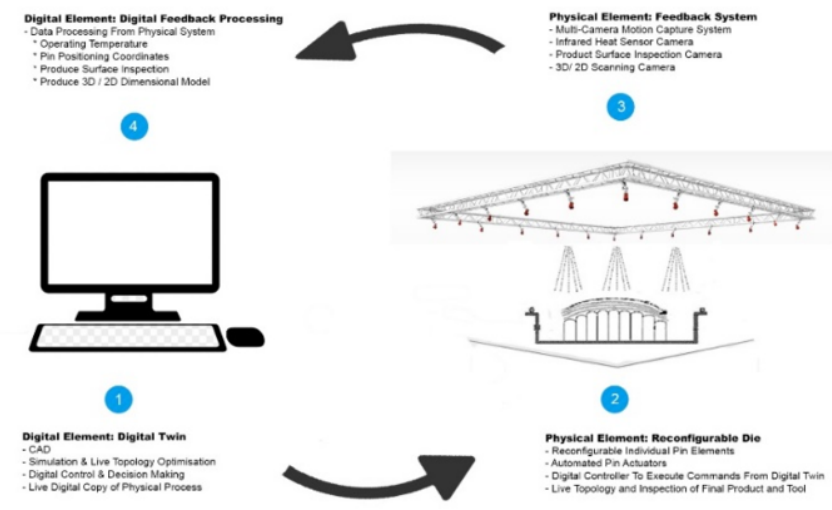

Figure 1. System Communication 
For the purpose of this study, the emulation model of the reconfigurable hot forming system will be the only system emulated and will send direct data to the digital twin without the need of modeling cameras, sensors and infrared equipment as required of the physical system. This simplifies the emulation model to be created as well as removes the potential of incorrect data and chances of error from said systems. At the construction level engineers are concerned with outlining and approximating the performance of the key operating zones in the system, thus if reduces as much as possible, it will aid in achieving a highly accurate emulation.

The aim of this paper is to model all the elements in the system in adequate depth so that execution and precision can be modeled to the upmost accuracy of the real system. At this level of emulation, the system precisely replicates all the operations and outcomes in the physical system. The amount of detail needed to achieve this for the complexity of the adopted system is quite high due to its complexity in nature with multiple individual parts operating simultaneously to achieve the desired outcome. The extraordinary need for the emulation model cycles to be as accurate as possible of that of the physical entity for both performance and correctness verification makes emulation velocity a vital influence in regulating the superiority of the system design.

In this study, the reconfigurable hot forming system utilised as the physical element of the digital twin system not only sends data back to the digital twin for the mirroring effect but also receives instructions from the digital twin, thus it is critical that the emulation model has the ability to emulate the system and simulate the system concurrently. I.e., receive instructions from the digital twin and act up on them immediately whilst sending data at the same time. Built in support for concurrency in this emulation model is the main characteristic that distinguishes an emulation model to a simple simulation model. The approach concurrency is replicated has a vast influence on both emulation model performance and modeling flexibility. The typical method of replicating concurrency is to utilise a dynamic scheduler that commands the implementation of user defined commands based on occurrences. This is identified as discrete occurrence imitation and is the highest accommodating method of simulating concurrency. The substitute to dynamic scheduling is rotation centered emulation. In this type of emulation all components, produce is evaluated once each rotational cycle is complete of which warrants all input proceedings to a component have their final value by the period the produce is executed.

\section{Virtual Emulation Model Development}

The software used to develop the emulation model is Simumatik3D, this software was built specifically with all key features tailored towards digital emulation of physical entities. It provides advantages such as easy and speed of building an emulation model, pre-existing large library of behavior of physical systems for ease of imitation of realworld physical systems.

\subsection{Phase 1-Analysis, Reverse Engineering and Development}

The involvedness and assortment of elements contained within the hot forming process supplied a challenging and interesting study. The hot forming capability is a process of 
which metal sheets are heated up to a desired temperature and stamped between two dies to form a desired shape.

The machine includes electric, pneumatic and hydraulic components, including several linear and rotational movements, conveyors, fixtures and robot. To control the process from an automation process it also includes a number of sensors, cameras and infrared sensors.

The effort started by exploring all the various machine movements and processes and developed a sequential description of the machine. Mechanical and electrical drawings were investigated to comprehend the machine construction and how different components work together. This is necessary to understand the machine functionality and aids the process of building each machine with Simumatiuk3D. The hot forming process that was analyzed and reverse engineered comprises a number of key elements:

- $\quad$ Hydraulic press with upper and lower dies (Forming station)

Heating elements (Furnace Heating Station)

- Material handling system:

- Robots (Robotic arms for handling)

- Conveyor belt

Reinforcing station

The first step in developing an emulation model of the hot forming capability and combining it with the digital twin system was to combine all the key elements of the forming process with the automated requirements to enable the digital twin to execute the principal function. By combining the hot forming system and fully automating the production line, figure 2 illustrates the system architecture developed. The layout of the machinery was designed to flow in the natural step by step manner of which hot forming process follows. The layout includes full automation from the material feeding stage until the final product is Produced.

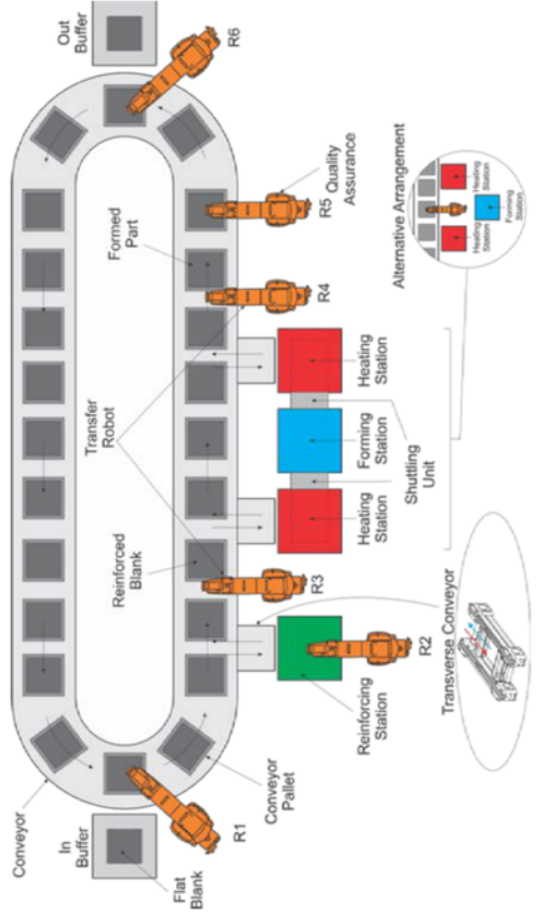

Figure 2. System Architecture

The hot forming capability is a process of which relies heavily on temperature and processing time for success. Blank sheet metals are formed in their soft state (austenite state) at high temperature and then quenched in the forming press between the female and male dies. The forming process follows the following steps:
(1) Buffer in
(2) Reinforcement
(Optional
(4) Transfer from furnace to forming press dependent on product)
(5) Quenching and forming in the cool press
(3) Heat to a desired temperature in furnace


(6) Transfer from forming press to furnace once more

(7) Quality Assurance

(8) Buffer out

Figure 3 illustrates the final outcome of the emulation model.

Phase 2 - Virtual Validation, Verification and Testing Having confirmed the validity and accuracy of the execution of the Digital Twin and development of the emulation model of the real-life physical system, it has become critical to validate and test

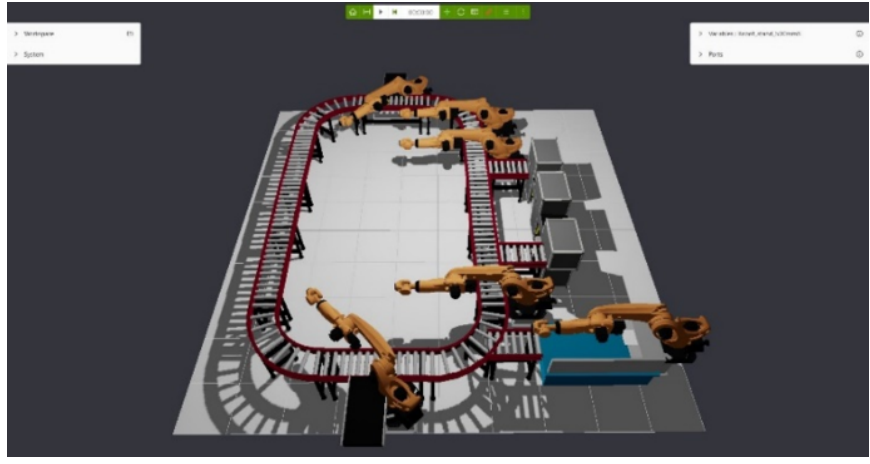

Figure 3. Emulation Model

the emulation model against the real-life physical machine to ensure the accuracy of the data provided from the digital emulation model to the Digital Twin system. The emulation model's purpose is to behave a like for like to the real physical entity, this validation and testing ensures and validates the case.

Once the Digital Twin was developed and tested of the accuracy of the control logic, the verification and validation of the emulation model is the final stage. Verification is described as the process of confirming if the emulation model was built right while validation confirm if the right emulation model was built. By way of explanation, the verification process centers on matching the components of an emulation model of the system with the description of what the requirements and capabilities of the emulation model were to be. Verification is a repetitive process designed at concluding whether the elements of each step in the execution of the emulation model achieves all the requirements imposed on it by the previous step and is conforming, consistent, and correct enough to support the next phase.

The validation stage on the other hand centers around the conformity between the observed behavior of elements of the physical system with the equivalent elements of an emulation model of the system and on establishing whether the variances are satisfactory given the planned use of the emulation model. If an acceptable conformity is not attained, the emulation model is fine-tuned to bring it in closer conformity with the observed behavior of the real physical system (or faults in observation/ investigation or reference models/analyses are detected and fixed).

In this validation, the emulation model elements validated are:

- $\quad$ Emulation model 1-1 alike the physical entity

- $\quad$ Each individual element accurate behavioral emulation of the physical system

- $\quad$ Conforming final produce

- $\quad$ Conforming functionality of each element

- $\quad$ Conforming behavior of the system as a unit

- $\quad$ Effective communication between emulation model and Digital Twin 
The VFAT methods employed in this study is a combination of:

- $\quad$ Historical Data Validation

- $\quad$ Face Validity

\subsubsection{Data Validation}

Having taken the above into account with cross comparing the data obtained from the physical system and the emulation model over 100 production runs and the model validated by technical operators, the emulation model was confirmed to be within the allowable tolerance hence successfully passed the validation and verification process.

There is no completely conclusive approach for validating the emulation model of the proposed system. If there were, then there might be no need for an emulation model in the first instance or even a digital twin as the system on the flip side would be immensely accurate with little to no variations between produces.

\section{Conclusion}

This paper presents the development and validation of the emulation model of the hot forming capability in which a Digital Twin has been built employing the emulation model as a replacement for the physical entity. The simulation tool - Simumatik3D was used to build the emulation model. The emulation model will be used to test the Digital Twin system before implementing the emulation model in conjunction with the Digital Twin in real life.

Once the emulation model is established, all the advantages of virtual development are witnessed in the development and validating phases. The time and cost devoted on building a virtual emulation model is paid off with the cutback of the real physical system time and costs in experimenting and production down time, and has even a progressive impact in the quality of the delivery. furthermore, all work accomplished with the Digital Twin influences considerably in sustainability, due to the energy consumption required for operating the Digital Twin is much lower than the real system as well as computing time being a lot faster than production time in real life. Virtual validation and confidence in the developed emulation model can be and should be attained before starting the implementation stage in a real factory. The primary conclusion that can be drawn from this study is that the objective was achieved successfully. The main objective was the development of an emulation model that operates at a 1:1 likeness for the hot forming capability to be utilised alongside and controlled via Digital Twin.

\section{References}

[1] Z. Liu, N. Suchold, C. Diedrich, Virtual Commissioning of Automated Systems, 2012,

[2] I. McGregor, The relationship between simulation and emulation, Proceedings of the Winter Simulation Conference, 2002, vol.1682, pp. 1683-1688.

[3] M. Oppelt, L. Urbas, integrated virtual commissioning an essential activity in the automation engineering process: From virtual commissioning to simulation supported engineering, IECON Proceedings (Industrial Electronics Conference), Institute of Electrical and Electronics Engineers Inc., 2014, pp. 2564-2570.

[4] "Hardware Emulation Workshop", DARPA ERI Summit, July 23-25 2018, San Francisco, CA, http://bit.ly/2xoL45n 